\title{
Lamb shift due to surface plasmon polariton modes
}

\author{
Qingqing Sun, ${ }^{1,2}$ M. Al-Amri, ${ }^{3,4}$ Ali Kamli, ${ }^{4,5}$ and M. Suhail Zubairy ${ }^{1,2}$ \\ ${ }^{1}$ Department of Physics and Institute of Quantum Studies, Texas A\&M University, College Station, Texas 77843-4242, USA \\ ${ }^{2}$ Texas A\&M University at Qatar, Education City, P.O. Box 23874, Doha, Qatar \\ ${ }^{3}$ The National Centre for Mathematics and Physics, KACST, P.O. Box 6086, Riyadh 11442, Saudi Arabia \\ ${ }^{4}$ Department of Physics, King Khalid University, Abha 61314, P.O. Box 9004, Saudi Arabia \\ ${ }^{5}$ Institute for Quantum Information Science, University of Calgary, Calgary, Alberta T2N 1N4, Canada
}

(Received 18 February 2008; published 2 June 2008)

\begin{abstract}
The Lamb shift of a hydrogen atom due to the surface plasmon polariton (SPP) modes is calculated and we observe both band edge and surface enhancement. The atom sits inside a thin metal slab which is sandwiched by two semi-infinite dielectrics. The SPP dispersion relation shows a band gap if the two dielectrics have different dielectric constants. We find the magnitude of the $2 P_{1 / 2}$ level shift increases rapidly when the level separation is close to the high band edge. But the low band edge enhancement can be overwhelmed by the decreasing trend of the shift. If the atom is placed close to either surface or if the slab is narrow, all the level shifts would be large because of the surface enhancement. An interesting feature is that the $2 P_{1 / 2}$ level shift can be larger than the $2 S$ level shift due to the nonisotropic fields.
\end{abstract}

DOI: $10.1103 /$ PhysRevA.77.062501

PACS number(s): 31.30.jf, 71.36.+c

\section{INTRODUCTION}

The discovery [1] and explanation [2] of the Lamb shift marked the beginning of modern quantum electrodynamics. This $2 S-2 P_{1 / 2}$ level difference arises from the coupling of the bound electron to the vacuum electromagnetic modes [3]. Since then the energy level shift has become one of the central themes in quantum optics. People have explored various modifications of the mode structure to get different shifts. Essentially these modifications come from some confined environments or periodic structures, for example, near a conducting plane [4], between parallel conducting planes $[5,6]$, inside a waveguide [7], inside a cavity [8], or inside a quantum well [9]. Various large, small, and negative Lamb shifts have been observed. Modification due to the band gap in the photonic crystal has also been studied, in one-, two- [10], and three-dimensional [11] systems.

In this paper we calculate the Lamb shift of a hydrogen atom due to the surface plasmon polariton (SPP) modes in a metallic slab sandwiched by two semi-infinite dielectrics. A surface plasmon is a longitudinal collective oscillation of the electrons near the surface between metal and dielectric. It can be coupled to an electromagnetic field and form a collective excitation, named as surface plasmon polariton. The field is an evanescent wave which propagates along the interface and decays into either side of the interface. SPP was first predicted by Ritchie while studying the problem of energy losses of fast electrons passing through thin metallic foils [12]. It was observed in later experiments by Powell and Swan [13]. Since then it has been explored in many fields including surface physics [14-16], electrochemistry [17], and biosensing [18]. The latest advance is to use SPP to build subwavelength photonic circuit $[19,20]$. For recent reviews, one is referred to [21].

On a planar surface between semi-infinite metal and dielectric, the SPP dispersion curve lies below the light line and asymptotically goes to the surface plasmon frequency in the nonretarded region. Therefore it is nonradiative due to the wave-vector mismatch. For a thin metal slab sandwiched by two semi-infinite dielectric regions, the SPP modes from two surfaces could interact with each other [22]. If the two dielectric constants are different there is a band gap in the dispersion relation.

We are interested in the Lamb shift induced by the SPP modes because the field can be concentrated both spatially and spectrally in this case. More parameters can be adjusted and giant shifts are expected. When the transition frequency $\omega_{21}$ lies inside the band gap, we calculate the $2 S$ and $2 P_{1 / 2}$ level shifts due to the modes of surface plasmon polaritons. For the $2 S$ level, it decreases with the plasmon frequency. For the $2 P_{1 / 2}$ level, the contribution from the high band always shows the band edge enhancement, but the band edge effect from the low band depends on the location. The level shifts are largely enhanced at the surfaces. $2 P_{1 / 2}$ level shift can be greater than $2 S$ level shift due to the nonisotropic fields.

\section{THEORY}

\section{A. Dispersion relations}

First we introduce the system. For simplicity, we assume the permittivities of the dielectric regions $\epsilon_{1}$ and $\epsilon_{2}$ to be frequency independent. The boundary of the metal slab are $z=0$ and $z=d$ planes. The dielectric function of the metal can be obtained by ignoring the absorption,

$$
\epsilon_{s}(\omega) \approx \epsilon_{b}-\frac{\omega_{p}^{2}}{\omega^{2}},
$$

where $\omega_{p}=\sqrt{\frac{n e^{2}}{\epsilon_{0} m^{*}}}$ is the bulk plasmon frequency, $\epsilon_{b} \approx 1$ includes the contribution from the bound electrons, $n$ is the number density of the free electrons, $m^{*}$ is the effective electron mass due to binding. This is a good approximation because mostly $1 / \omega$ is on the order of $10^{-16} \mathrm{~s}$ and much smaller than the typical relaxation time $10^{-14} \mathrm{~s}$ [23]. For $\omega$ $<\omega_{p}$, we have negative permittivity. So the wave vector in- 


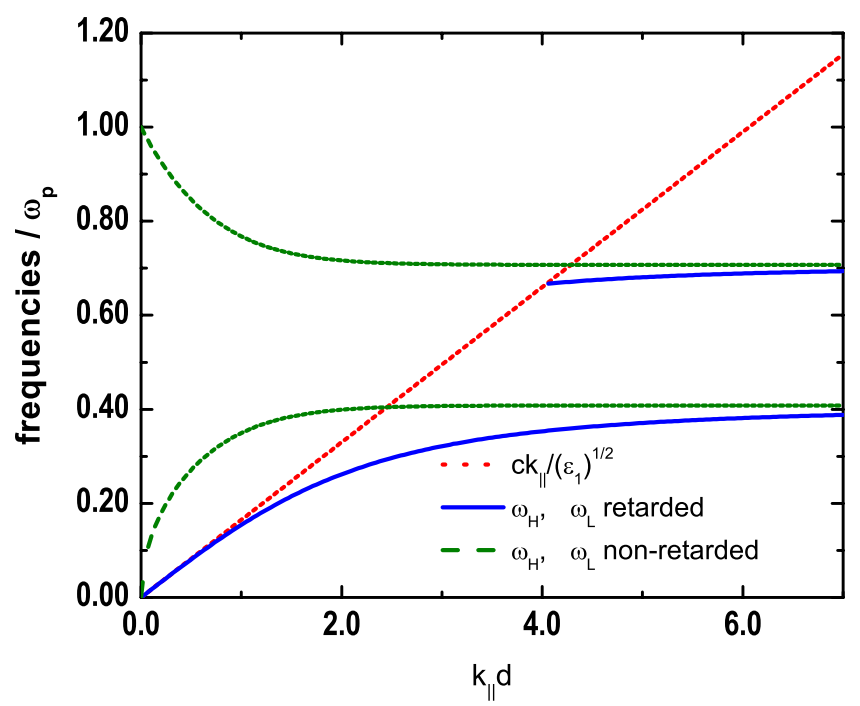

FIG. 1. (Color online) Dispersion relations of the SPP mode when the two dielectrics are unsymmetrical. The solid curve is the retarded dispersion. From one line at small wave vector, it changes into two lines discontinuously and shows a band gap. The subscript $H$ means the high band, and $L$ means the low band. Both lines start from the light dispersion line which is the dotted curve. The dispersion under nonretarded approximation is shown in dashed lines. Asymptotically they go to the same limits as the retarded dispersion.

side the slab $k_{s}$ satisfies $k_{s}^{2}=\epsilon_{s} \frac{\omega^{2}}{c^{2}}<0$, which means no propagation mode in the metal.

SPP modes decay into either side of the surface, i.e., they have imaginary wave vectors in the $z$ direction. Without loss of generality, we can assume the wave vectors in the three regions to be $\vec{k}_{1}=\left(\vec{k}_{\|},-i \beta_{1}\right), \vec{k}_{s+}=\left(\vec{k}_{\|}, i \beta_{s}\right), \vec{k}_{s-}=\left(\vec{k}_{\|},-i \beta_{s}\right), \vec{k}_{2}$ $=\left(\vec{k}_{\|}, i \beta_{2}\right)$. Here $\vec{k}_{\|}$is the wave vector projection on the $x y$ plane and $\beta_{j}(j=1,2, s)$ is the $z$ component. From the boundary conditions we know there is no TE wave, and the dispersion relation for the TM wave is [24]

$$
\tanh \left(\beta_{s} d\right)=-\frac{y_{1}+y_{2}}{1+y_{1} y_{2}}
$$

where $y_{j}=\frac{\epsilon_{s} \beta_{j}}{\beta_{s} \epsilon_{j}}$. If $\epsilon_{1} \neq \epsilon_{2}$, this transcendental equation has one solution at small $k_{\|}$and two solutions when $k_{\|}$is large. For example, when the width $d=35 \mathrm{~nm}, \epsilon_{1}=5, \epsilon_{2}=1$, we demonstrate the dispersion curve in the solid lines of Fig. 1. The high curve starts from some point on the light dispersion line instead of the origin, because those modes over the light line propagate in region 1 .

For large wave vectors, the frequency goes to a finite value asymptotically so that $k_{\|}^{2} \gg \omega^{2} \epsilon_{j} / c^{2}$ and $\beta_{j} \approx k_{\|}$. On substituting this and the dielectric function $\epsilon_{s}(\omega) \approx 1-\frac{\omega_{p}^{2}}{\omega^{2}}$ into the dispersion relation,

$$
\omega=\frac{\omega_{p}}{\sqrt{1+\frac{\left(\epsilon_{1}+\epsilon_{2}\right) \pm \sqrt{\left(\epsilon_{1}+\epsilon_{2}\right)^{2}-4 \tanh ^{2}\left(k_{\|} d\right) \epsilon_{1} \epsilon_{2}}}{2 \tanh \left(k_{\|} d\right)}}} .
$$

This is the explicit dispersion relation. It is valid for large wave vectors where the phase velocity is much smaller than $c$. So it is a nonretarded approximation.

When $k_{\|} \rightarrow \infty$ we find two limiting frequencies $\omega_{i}=\frac{\omega_{p}}{\sqrt{1+\epsilon_{i}}}$ $(i=1,2)$. They are equal if $\epsilon_{1}=\epsilon_{2}$. But for asymmetrical dielectrics, the difference between these frequencies causes the band gap. As a comparison to the exact dispersion, the dispersion relation under nonretarded approximation is shown in Fig. 1 as dashed lines.

\section{B. Field quantization}

Once we have the dispersion relation, we can quantize the field following the usual procedures $[25,26]$. First we write down the total electric field,

$$
\vec{E}(\vec{r}, t)=\sum_{\lambda} \int d^{2} \vec{k}_{\|}\left[\vec{E}_{0, \lambda}\left(\vec{k}_{\|}, z\right) a_{\lambda}\left(\vec{k}_{\|}\right) e^{i\left[\vec{k}_{\|} \cdot \vec{r}_{\|}-\omega_{\lambda}\left(\vec{k}_{\|}\right) t\right]}+\text { H.c. }\right],
$$

where $\lambda$ is the band index. Each $k_{\|}$could correspond to two frequencies, one in the high band and one in the low band. There is no polarization index since only TM modes exist. We know the field for one mode can be written as

$$
\vec{E}_{0, \lambda}\left(\vec{k}_{\|}, z\right)=C_{\lambda}\left(k_{\|}\right)\left[\hat{r}_{\|} F_{\lambda}\left(k_{\|}, z\right)+\hat{z} G_{\lambda}\left(k_{\|}, z\right)\right],
$$

where $C_{\lambda}, F_{\lambda}, G_{\lambda}$ do not depend on the direction of $\vec{k}_{\|}$due to symmetry. Notice that the piecewise functions $F_{\lambda}\left(k_{\|}, z\right)$ and $G_{\lambda}\left(k_{\|}, z\right)$ have the $z$ dependence. We can choose the coefficient of $F_{\lambda}\left(k_{\|}, z\right)$ in region 1 . Then all the other $F_{\lambda}, G_{\lambda}$ expressions follow from the boundary conditions. In region 1 $(z<0)$

$$
\begin{gathered}
F_{\lambda}\left(k_{\|}, z\right)=e^{\beta_{1} z}, \\
G_{\lambda}\left(k_{\|}, z\right)=-i \frac{k_{\|}}{\beta_{1}} e^{\beta_{1} z} .
\end{gathered}
$$

In the metal slab region $(0<z<d)$,

$$
\begin{gathered}
F_{\lambda}\left(k_{\|}, z\right)=M e^{-\beta_{s} z}+N e^{\beta_{s}(z-d)}, \\
G_{\lambda}\left(k_{\|}, z\right)=i \frac{k_{\|}}{\beta_{s}}\left[M e^{-\beta_{s} z}-N e^{\beta_{s}(z-d)}\right] .
\end{gathered}
$$

In region $3(z>d)$,

$$
\begin{gathered}
F_{\lambda}\left(k_{\|}, z\right)=e^{-\beta_{2}(z-d)} R, \\
G_{\lambda}\left(k_{\|}, z\right)=i \frac{k_{\|}}{\beta_{2}} e^{-\beta_{2}(z-d)} R,
\end{gathered}
$$

where

$$
M=-\frac{1}{2} \frac{1-y_{1}}{y_{1}}
$$




$$
\begin{aligned}
& N=-M \frac{1-y_{2}}{1+y_{2}} e^{-\beta_{s} d}, \\
& R=2 M \frac{y_{2}}{1+y_{2}} e^{-\beta_{s} d} .
\end{aligned}
$$

It follows, on comparing the Hamiltonian for this dispersive medium [27] and the classical Hamiltonian, that we obtain the normalization constant

$$
C_{\lambda}\left(k_{\|}\right)=\left(\frac{A}{4 \pi^{2}}\right)^{1 / 2}\left\{\frac{\hbar \omega_{\lambda}\left(k_{\|}\right)}{4 \pi^{2} \epsilon_{0}\left\{D+\left[\omega_{\lambda}^{2}\left(k_{\|}\right) / c^{2}\right] S\right\}}\right\}^{1 / 2},
$$

in which $A$ is the area with

$$
\begin{aligned}
D= & \frac{\epsilon_{1}}{2 \beta_{1}^{3}}\left(\beta_{1}^{2}+k_{\|}^{2}\right)+\frac{\epsilon_{2}}{2 \beta_{2}^{3}}\left(\beta_{2}^{2}+k_{\|}^{2}\right) R^{2}+\frac{\partial}{\partial \omega}\left(\omega \epsilon_{s}\right) \\
& \times\left[\left(M^{2}+N^{2}\right) \frac{\beta_{s}^{2}+k_{\|}^{2}}{\beta_{s}^{2}} \frac{\sinh \left(\beta_{s} d\right)}{\beta_{s} d}+2 M N \frac{\beta_{s}^{2}-k_{\|}^{2}}{\beta_{s}^{2}}\right] e^{-\beta_{s} d} d, \\
S= & \frac{\epsilon_{1}^{2}}{2 \beta_{1}^{3}}+\frac{\epsilon_{2}^{2}}{2 \beta_{2}^{3}} R^{2}+\frac{\epsilon_{s}^{2}}{\beta_{s}^{2}}\left[\left(M^{2}+N^{2}\right) \frac{\sinh \left(\beta_{s} d\right)}{\beta_{s} d}-2 M N\right] e^{-\beta_{s} d} d .
\end{aligned}
$$

\section{Lamb shift}

Lamb shift is the level difference between $2 S$ and $2 P_{1 / 2}$ and shift when an atom interacts with the vacuum modes. Here the modes are modified and we borrow this term. The Hamiltonian for an atom inside the slab can be written as

$$
\mathcal{H}=\mathcal{H}_{\text {atom }}+\mathcal{H}_{\text {field }}+\mathcal{H}_{\text {int }}+\mathcal{H}_{\text {ct }},
$$

where the interaction Hamiltonian

$$
\mathcal{H}_{\text {int }}=-\frac{e}{m} \vec{p} \cdot \vec{A}(\vec{r})+\frac{e^{2}}{2 m} \vec{A}^{2}(\vec{r}) .
$$

$\vec{A}$ is the vector potential operator with the coefficient $\vec{A}_{0, \lambda}^{*}\left(\vec{k}_{\|}, z\right)=i \vec{E}_{0, \lambda}^{*}\left(\vec{k}_{\|}, z\right) / \omega_{\lambda}\left(k_{\|}\right) . \mathcal{H}_{c t}$ is the counter term since the measured electron mass has already included the fluctuation contribution. Second-order perturbation theory gives the general expression for the shift of level $|l\rangle$ as in [3],

$$
\Delta E_{l}=\sum_{n} \sum_{\vec{k}} \frac{\left|\left\langle n, 1_{\vec{k}}|-(e / m) \vec{p} \cdot \vec{A}(\vec{r})| l, v a c\right\rangle\right|^{2}}{E_{l}-E_{n}-\hbar \omega_{k}}+\Delta E_{c t} .
$$

The $\frac{e^{2}}{2 m} \vec{A}^{2}(\vec{r})$ term is ignored because it contributes the same shift to each level. The mass renormalization term $\Delta E_{c t}$ can be obtained by taking $E_{l}=E_{n}$ in the first term.

With the knowledge of the electric field and the retarded dispersion relation for the SPP modes, we can calculate their contribution to the shift of level $|2\rangle$.

$$
\begin{aligned}
\Delta E_{2}(z)= & \frac{2 \pi}{3}\left(\frac{e}{m}\right)^{2} \sum_{n=0}^{\infty} \sum_{\lambda}\left|\vec{p}_{2 n}\right|^{2} \int_{0}^{\Lambda} k_{\|} d k_{\|} \\
& \times \frac{4 \pi^{2}}{A} \frac{C_{\lambda}^{2}\left(k_{\|}\right)\left[\left|F_{\lambda}\left(k_{\|}, z\right)\right|^{2}+\left|G_{\lambda}\left(k_{\|}, z\right)\right|^{2}\right]}{\omega_{\lambda}^{2}\left(k_{\|}\right)} \\
& \times\left[\frac{1}{E_{2}-E_{n}-\hbar \omega_{\lambda}\left(k_{\|}\right)}-\frac{1}{-\hbar \omega_{\lambda}\left(k_{\|}\right)}\right] .
\end{aligned}
$$

For simplicity we have ignored the relativistic contribution by taking a conventional cutoff wave vector $\Lambda=m c / \hbar$ [2]. This cutoff value is not important because the integration converges far before $k$ reaches $\Lambda$. There are also contributions from some other modes, for example, when $\omega<\omega_{p}$ there are propagation modes in the dielectrics which are evanescent in the metal, and when $\omega>\omega_{p}$ there are modes propagational in both the metal and dielectrics. Here we concentrate on the contributions from non-propagating SPP modes only. This contribution is only dominant close to the surfaces. At other locations all the modes need to be included to find the total level shift.

\section{NUMERICAL RESULTS}

The level shift of a hydrogen atom due to SPP modes can be calculated with the knowledge of all the momentum matrix elements [28], both discrete and continuous. First we want to see the effect of the band edge. The level separation is fixed and cannot scan across the band gap. So we move the band gap instead, which can be achieved by using metals with different $n$ and therefore different plasmon frequencies. Under the parameters $d=35 \mathrm{~nm}$, the atom location $z$ $=5 \mathrm{~nm}, \epsilon_{1}=5, \quad \epsilon_{2}=1, n$ from $1.72 \times 10^{29} / \mathrm{m}^{3}$ to 4.56 $\times 10^{29} / \mathrm{m}^{3}$ we have calculated the shift for both $2 S$ and $2 P_{1 / 2}$ levels. The contribution from the low band and the high band are shown separately in Fig. 2. In most of the curves the magnitudes decrease with the plasmon frequency, which can be seen from the denominator of the level shift expression. The only exception is the low band contribution to $2 P_{1 / 2}$ level which decreases first and then increases rapidly at large plasmon frequency. It is reasonable because a larger $\omega_{p}$ moves the whole band gap up and makes the low band edge closer to the level separation $\omega_{21}$. Similarly at a small $\omega_{p}$ the high band edge is close to the level separation. So the high band contribution to the $2 P_{1 / 2}$ level rises up quickly. This band edge effect does not exist in the $2 S$ level shift because there is no transition from $2 S$ to $1 S$. For the allowed $2 P_{1 / 2}$ $-1 S$ transition, the high band frequency $\omega_{H}\left(k_{\|}\right)>\omega_{21}$. From the last line of Eq. (16) we can see its contribution to $\Delta E_{2}$ is negative, as shown in Fig. 2(d).

In Fig. 2, the low band contribution is much larger than the high band contribution so we draw them separately. In order to see their effect together we change the location of the atom to $z=15 \mathrm{~nm}$. As one can see from Fig. 3, the contributions from the two bands are on the same order in this case. However, the low band contribution no longer increases at the band edge. This can be explained from Fig. 1. Since the low band edge is reached at the large wave vector limit, for which the exponential term $e^{-\beta_{s} z}$ in Eq. (7) would 

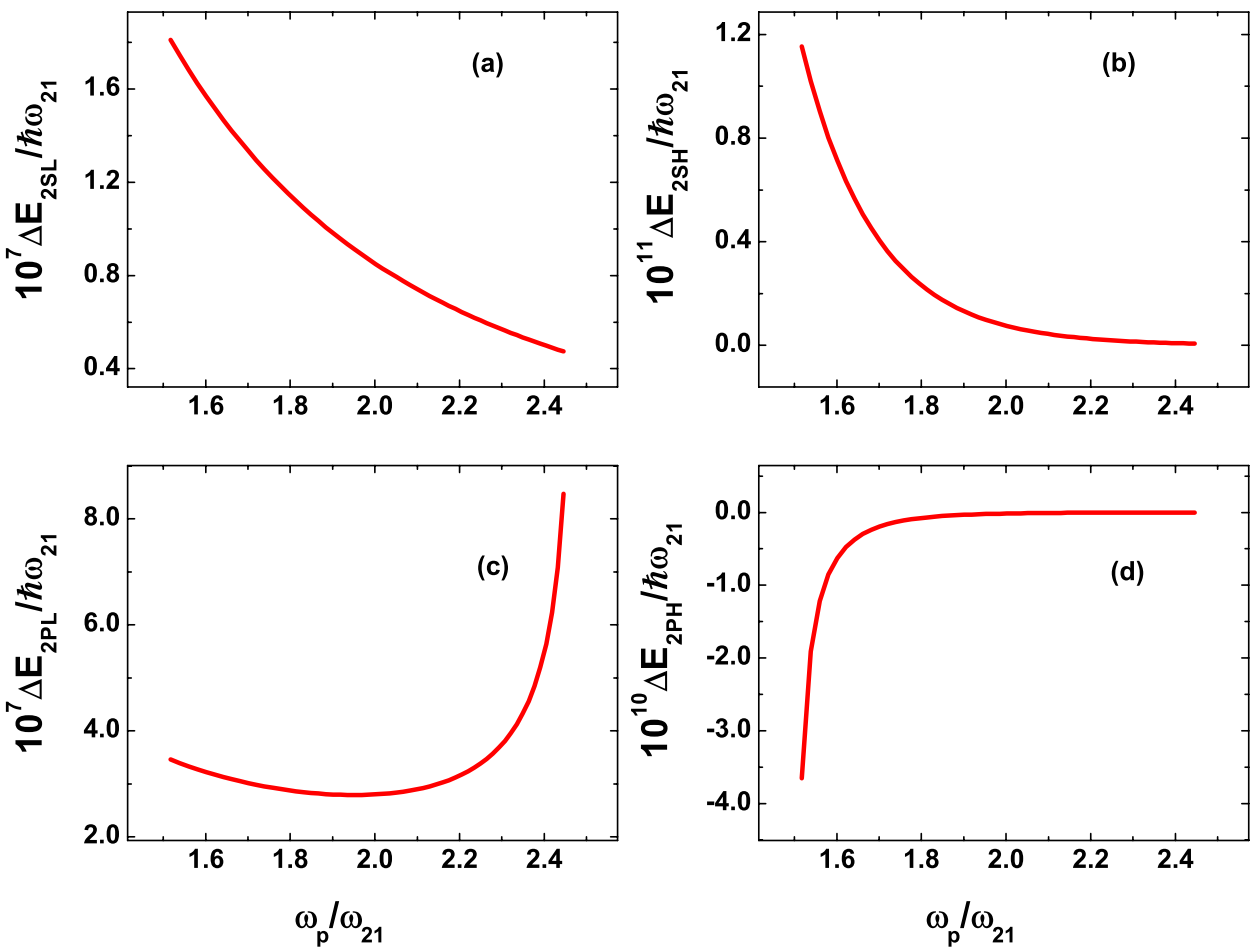

FIG. 2. (Color online) (a) Low band contribution to the $2 S$ level shift. (b) High band contribution to the $2 S$ level shift. (c) Low band contribution to the $2 P_{1 / 2}$ level shift. (d) High band contribution to the $2 P_{1 / 2}$ level shift. For most curves the magnitude decreases with $\omega_{p}$ except the rapid increase at the low band edge in (c). The magnitude is also large at the high band edge in (d). be too small at $z=15 \mathrm{~nm}$. So the band edge enhancement cannot compete with the decreasing trend. The high band contribution still shows the enhancement because the edge is at the starting point of the band where the wave vector is the smallest. The total contribution to the $2 P_{1 / 2}$ level shift changes in an interesting way. It first increases quickly from the large negative value at the high band edge, and then after reaching a maximum positive value it starts decreasing slowly.

We have noticed that the change of the atom location affects the level shift. For a fixed $n=3 \times 10^{29} / \mathrm{m}^{3}$ we sweep the atom from one side to another. From Fig. 4 the level shifts have large magnitudes at both sides. This is the surface enhancement since the fields decrease exponentially into the slab. The level shifts at side 1 almost totally come from the low band. At side 2 they come from the high band, so $\Delta E_{2 P}<0$ as we explained before. During the movement the low band contribution decreases quickly and the high band contribution increases quickly. The slab is not thin enough to see a strong interaction between the SPP modes.

If the atom is put in the dielectric but close to the slab, there is also surface enhancement to the SPP contribution as shown in Fig. 4. In this case the local field effect must be included. Following the real cavity model [29], we get the local field factor

$$
R_{G L}(\omega)=\frac{3 \epsilon(\omega)}{2 \epsilon(\omega)+1},
$$

for the electric field. In either dielectric it is a constant because we assume the $\epsilon$ to be frequency independent.

We have also considered the influence of the slab width. In Fig. 5 the width $d$ changes from $30 \mathrm{~nm}$ to $50 \mathrm{~nm}$ and the atom is always put at the center. The SPP contribution to both level shifts decrease to 0 at thick width, which is again because of the exponentially decay from the surface.

\section{DISCUSSIONS AND CONCLUSION}

This system has many adjustable parameters to control the level shifts. For example, close to the surface the shifts could be larger than the values in the vacuum. Another interesting feature is that $2 P_{1 / 2}$ level shift could be larger than the $2 S$ level shift. It should be noted that in vacuum the $2 P_{1 / 2}$ level shift is much smaller than the $2 S$ level shift and it is explained heuristically that the level shift should be proportional to the square value of the wave function at the center due to the isotropic fluctuations. In this system the fields are no longer isotropic as in the vacuum, so the $2 P_{1 / 2}$ level shift can have large values.

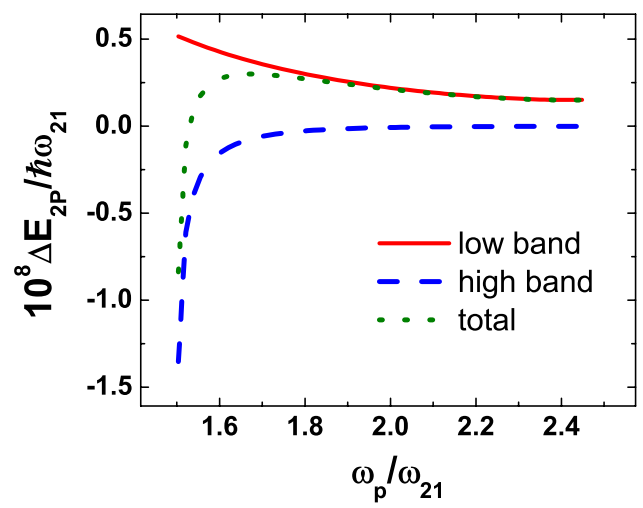

FIG. 3. (Color online) Contribution to the $2 P_{1 / 2}$ level shift at $z=15 \mathrm{~nm}$. The low band edge enhancement is overwhelmed by the decreasing trend due to the larger plasmon frequency. 

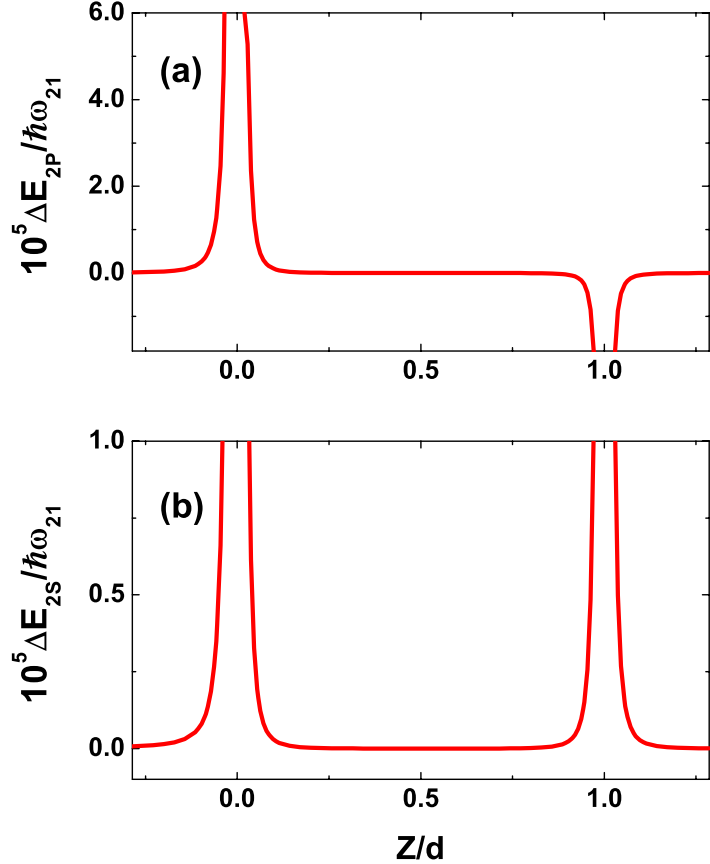

FIG. 4. (Color online) Contribution to the level shifts for different locations. They increase rapidly close to the surface. At side 1 the contribution is mainly from the low band, while at side 2 from the high band.

During the investigation we adopt the free electron gas model, which requires metals with no interband transition up to $\omega_{p} / \sqrt{2}$. Another condition is the slab width needs to be much larger than the electron mean free path [30]. The number densities we use are slightly high among the metals [31]. So the typical mean free path at room temperature should be around or smaller than $10 \mathrm{~nm}$ [32], which means the $35 \mathrm{~nm}$ width is barely enough to meet the condition. If we chose the width to be $100 \mathrm{~nm}$ then all the level shifts would have similar trends but with smaller numbers.

In conclusion, for a hydrogen atom in a metal slab, we have shown the contribution to the level shift due to the surface plasmon polaritons. For the $2 P_{1 / 2}-1 S$ transition when the level separation is close to the high band edge the magnitude of the level shift increases rapidly. But at the low band edge the enhancement could be overwhelmed by the

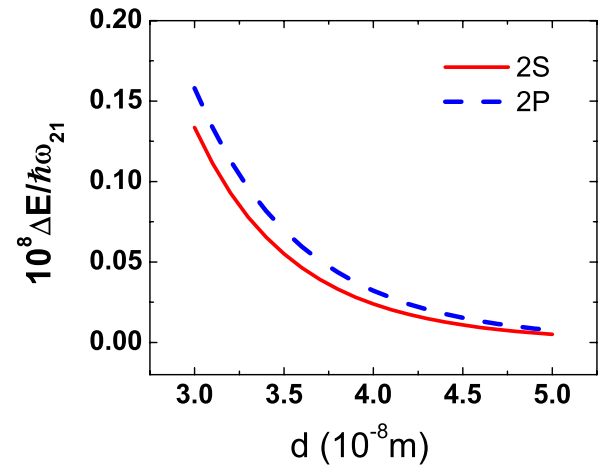

FIG. 5. (Color online) Contribution to both level shifts decrease to zero when the slab become too thick. This is another example of the surface effect.

decreasing trend. Close to the surface all the level shifts magnitude increase because of the larger fields. In locations close to side 1 the low band contributes mostly, while close to side 2 the high band contributes the most. Also the level shift is larger for a narrower slab. By changing the parameters we can have various values for the level shifts, even larger than the vacuum values. The $2 P_{1 / 2}$ level shift could even be greater than $2 S$ due to the nonisotropic fields, which means a negative contribution to the Lamb shift.

Experimentally it is not easy to measure the location and Lamb shift of an atom inside metal. An alternative scheme is to consider an impurity or defect in a doped semiconductor, which behaves more like a conductor and also supports surface plasmon polaritons. Similar to our treatment one can use a hydrogenic color center model to find the level shift. In this case the local field effect should be taken into consideration. Another way is to measure the level shift outside of the metal since there an atom can be put in a location accurately and measured easily.

\section{ACKNOWLEDGMENTS}

This research is supported by grants from the King Abdul Aziz City of Science and Technology (KACST) and the Qatar National Research Fund (QNRF). Ali Kamli acknowledges support from iCore.
[1] W. E. Lamb, Jr. and R. C. Retherford, Phys. Rev. 72, 241 (1947).

[2] H. A. Bethe, Phys. Rev. 72, 339 (1947).

[3] P. W. Milonni, The Quantum Vacuum: An Introduction to Quantum Electrodynamics (Academic, San Diego, 1993).

[4] G. Barton, J. Phys. B 7, 2134 (1974).

[5] G. Barton, Proc. R. Soc. London, Ser. A 410, 141 (1987).

[6] M. Marrocco, M. Weidinger, R. T. Sang, and H. Walther, Phys. Rev. Lett. 81, 5784 (1998).

[7] P. Horak, P. Domokos, and H. Ritsch, Europhys. Lett. 61, 459 (2003).

[8] D. Kleppner, Phys. Rev. Lett. 47, 233 (1981).
[9] A. L. Ivanov and H. Haug, Jpn. J. Appl. Phys., Suppl. 34, 143 (1995).

[10] S. John and J. Wang, Phys. Rev. Lett. 64, 2418 (1990); Phys. Rev. B 43, 12772 (1991); S. John and T. Quang, Phys. Rev. Lett. 74, 3419 (1995); S. John and T. Quang, ibid. 76, 1320 (1996); S. John and T. Quang, ibid. 78, 1888 (1997).

[11] S.-Y. Zhu, Y. Yang, H. Chen, H. Zheng, and M. S. Zubairy, Phys. Rev. Lett. 84, 2136 (2000).

[12] R. H. Ritchie, Phys. Rev. 106, 874 (1957).

[13] C. J. Powell and J. B. Swan, Phys. Rev. 115, 869 (1959); 116, 81 (1959).

[14] J. Schmit and A. A. Lucas, Solid State Commun. 11, 415 
(1972).

[15] D. C. Langreth and J. P. Perdew, Phys. Rev. B 15, 2884 (1977).

[16] P. M. Echenique, R. H. Ritchie, N. Barberan, and J. Inkson, Phys. Rev. B 23, 6486 (1981).

[17] W. Knoll, Annu. Rev. Phys. Chem. 49, 569 (1998).

[18] M. Malmqvist, Nature (London) 361, 186 (1993).

[19] B. Hecht, H. Bielefeldt, L. Novotny, Y. Inouye, and D. W. Pohl, Phys. Rev. Lett. 77, 1889 (1996).

[20] J. Pendry, Science 285, 1687 (1999).

[21] W. L. Barnes, A. Dereux, and T. W. Ebbesen, Nature (London) 424, 824 (2003); A. V. Zayats and I. I. Smolyaninov, J. Opt. A, Pure Appl. Opt. 5, S16 (2003); J. M. Pitarke, V. M. Silkin, E. V. Chulkov, and P. M. Echenique, Rep. Prog. Phys. 70, 1 (2007).

[22] E. A. Stern and R. A. Ferrell, Phys. Rev. 120, 130 (1960).

[23] S. A. Maier, Plasmonics: Fundamentals and Applications
(Springer, New York, 2007).

[24] P. Ahlqvist, Phys. Scr. 26, 217 (1982).

[25] A. Kamli and M. Babiker, Phys. Rev. A 62, 043804 (2000).

[26] J. Hakami, A. Kamli, and M. Al-Amri, Opt. Commun. 279, 112 (2007).

[27] L. D. Landau, E. M. Lifshitz, and L. P. Pitaevskii, Electrodynamics of Continuous Media (Elsevier, Oxford, 2006).

[28] H. A. Bethe, L. M. Brown, and J. R. Stehn, Phys. Rev. 77, 370 (1950).

[29] R. J. Glauber and M. Lewenstein, Phys. Rev. A 43, 467 (1991).

[30] E. N. Economou, Phys. Rev. 182, 539 (1969).

[31] N. W. Ashcroft and N. D. Mermin, Solid State Physics (Saunders College Publishing, Philadelphia, 1976).

[32] W. Zhang, S. H. Brongersma, O. Richard, B. Brijs, R. Palmans, L. Froyen, and K. Maex, Microelectron. Eng. 76, 146 (2004). 